\title{
Thermodynamics of Formation of Porous Polymeric Membrane by Phase Separation Method IV. Pore Formation by Contacting Secondary Particles: Computer Simulation Experiments
}

\author{
Kenji Kamide, Hideki Iusma, ${ }^{*}$ and Akira KataoKA* \\ Laboratory of Clothing, Faculty of Education, Kumamoto University, \\ Kurokami 2-40-1, Kumamoto 860, Japan \\ * Fundamental Research Laboratory of Natural \& Synthetic Polymers, \\ Asahi Chemical Industry Co., Ltd., \\ 11-7 Hacchonawate, Takatsuki, Osaka 569, Japan
}

(Received October 20, 1993)

\begin{abstract}
An attempt was made to investigate the process of forming porous polymeric membrane by computer simulation experiments based on Monte Carlo method. In this approach secondary particles (i.e., polymer-rich phase; referred to as polymer particles) and vacant particles (i.e., polymer-lean phase) are assumed to have the same radius $S_{2}$ and the number ratio of those particles is predetermined by two-phase volume ratio $R\left(\equiv V_{(1)} / V_{(2)} ; V_{(1)}\right.$ and $V_{(2)}$ are volumes of polymer-lean and -rich phases, respectively). These particles are arranged randomly on a hypothetical hexagonally closest packing lattice plane and all pores consisting of $x$ vacant particles, which are referred to as "vacant-particle pores," are counted one by one to give probability of appearance of $N_{\mathrm{P}}$ vacant-particle pores in unit area of membrane, $Q\left(N_{\mathrm{P}}\right)$, from which expected value of $N_{\mathrm{P}}, \overline{N_{\mathrm{P}}}$ are determined. Probability $P(x)$ that a given pore contains $x$ vacant particles and the average of number of vacant particles in one pore, $\bar{x}$ are also determined. In the range of $R \leq 0.4$, the theory on pore size distribution, proposed by Iijima, Matsuda, and Kamide in a previous paper, gives the same curves of $P(x), N_{\mathrm{P}}$, and $\bar{x}$ (accordingly, number-average pore radius of wet membrane, $\overline{r_{\text {Wet, } 1}}$ as those by the simulation, meaning that the theory holds its validity with respect to pore size distribution $N(r)\left(r\right.$, radius of pore) and $N_{\mathrm{p}}$. Morphology and $P(x)$ of the piled plane are shown to be practically the same as those of a hypothetical thin plane directly formed at an apparent phase volume ratio $R_{\mathrm{A}}$, evaluated from the number ratio of polymer particles and vacant particles on the surface of the piled plane by taking into consideration of overlapping. It is ascertained that computer experiments can produce membranes with morphology very similar to actual membranes and we can evaluate, by computer simulation, accurately the morphology and pore size distribution using $R_{\mathrm{A}}$, in place of $R$.

KEY WORDS Particle Growth Concept / Phase Separation / Porous Polymeric Membrane / Monte Carlo Method / Computer Simulation Experiment / Hexagonal Lattice / Pore Size Distribution /
\end{abstract}

The formation of supermolecular structure, such as crystallinity; orientation of molecular chains; size, shape, and volume fraction of both primary and secondary particles; porosity; pore size distribution; pore shape, of polymer membranes by the solvent-casting method is without exception performed through the phase separation phenomena caused by mixing of the polymer solution with non- solvent (i.e., coagulant). The detailed steps are illustrated in Figure 1. In the previous papers, ${ }^{1-3}$ we reported some theoretical studies, together with computer and actual experiments, on the generation of primary particles (Figures $1 \mathrm{~b}-1 \mathrm{~d}),{ }^{1}$ the amalgamation of the primary particles to secondary particles (Figures 1d-1f), ${ }^{2}$ and the steps of formation of porous structures by mutual contact of the 


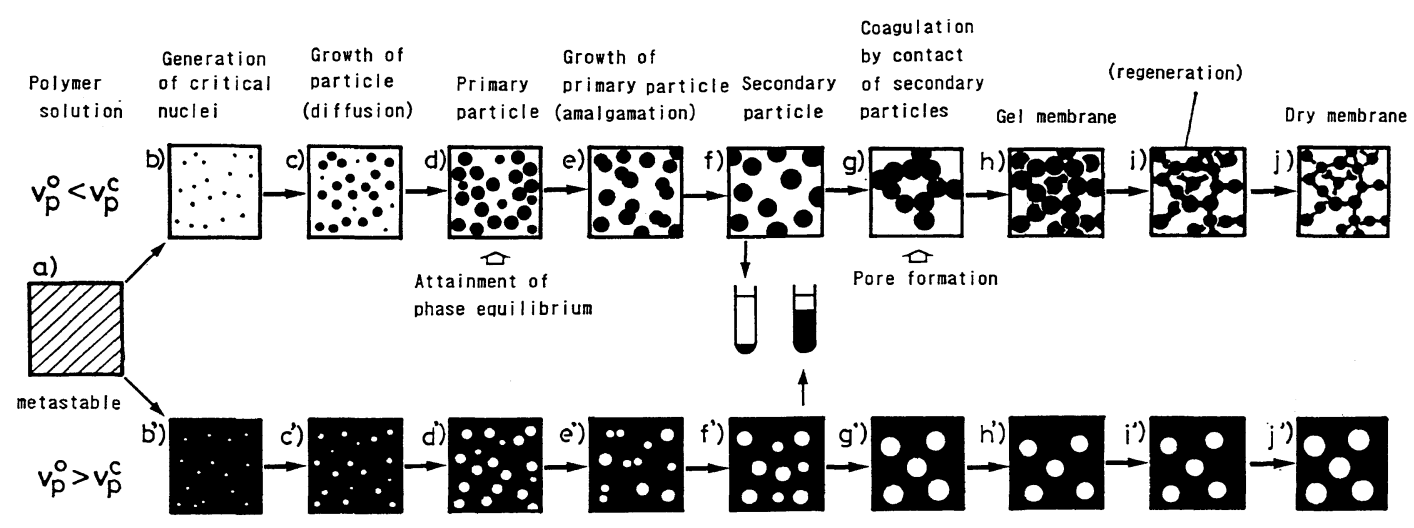

Figure 1. Elementary steps in porous polymeric membrane formation by the phase separation method: $v_{\mathrm{p}}^{0}$, polymer volume fraction of the solution when the phase separation occurs; $v_{\mathrm{p}}^{\mathrm{c}}$, polymer volume fraction of critical solution point.

secondary particles of polymer-rich phase (referred to as "polymer particles") (Figures $1 \mathrm{f}-1 \mathrm{k}){ }^{3}$ For the latter steps we derived an equation of the pore size distribution $N(r)$ ( $r$, radius of pore) or the probability $P(x)$ that a given pore contains $x$ hypothetical particles of polymer-lean phase ( $x$, number of hypothetical particles of polymer-lean phase constituting a single pore; referred to as "vacant particles") from the number of ways of partitioning polymer particles with radius $S_{2}$ and vacant particles on the hexagonal close-packed lattice. Note that the composition of these two kinds of the particles is determined unambiguously by the two-phase volume ratio $R\left(\equiv V_{(1)} / V_{(2)}\right.$; $V_{(1)}$ and $V_{(2)}$ are volumes of polymer-lean and -rich phases, respectively). $N(r)$ calculated by the equation (see, eq 24 of ref 3 ) using experimental data on $S_{2}$, porosity determined by the apparent density method, ${ }^{3} \operatorname{Pr}\left(\mathrm{d}_{3}\right)$ and that by electron micrographic method, ${ }^{4} \operatorname{Pr}\left(\mathrm{d}_{4}\right)$ for actual membranes coincided well with the pore size distribution $N(r)_{\mathrm{EM}}$ directly evaluated by electron micrographic method ${ }^{5}$ on dry membranes when the effect of contraction of thin gel membranes on $R$ in the equation was adequately considered. ${ }^{3}$ However, the above theory has the following short points: (1) the arrangement of only polymer particles located at the nearest neighbor positions to a given polymer particle on the hexagonal close-packed lattice was taken into account in the calculation of $N(r)$ and (2) all the pores consisting of vacant particles (referred to as "vacant-particle pores") were assumed to be perfectly circular. The improvement of the theory seems very tedious and difficult. An effective remedy to overcome these short points in the theory is usage of computer for simulation of the structure formation under the experimentally accessible conditions.

In this article, an attempt was made, by carrying out the computer simulation of arranging of the polymer particles and vacant particles on the hexagonal close-packed lattice in random manner, (1) to evaluate the probability of appearance of $N_{\mathrm{P}}$ vacant-particle pores in unit area of membrane surface for a given combination of $R$ and $S_{2}$ under the random distribution of particles, $Q\left(N_{\mathrm{P}}\right),(2)$ to clarify effects of $R$ on pore characteristics of membrane like $P(x)$, number of vacant-particle pores in unit area of membrane by simulation, $\overline{N_{\mathrm{P}}}$, and number-average pore radius for wet membrane, $\overline{r_{\text {Wet }, 1}},(3)$ to disclose the applicability limit of the equation of $N(r)$ in the theory proposed by Iijima, Matsuda, and Kamide in the previous paper (simply referred to as IMK theory), ${ }^{3}$ (4) to compare the supermolecular structures estimated by the computer simula- 
tion with those of cellulose membranes, and (5) to simulate the effect of the structural contraction during the coagulation step (Figures 1f to $1 \mathrm{~h}$ ) on the pore characteristics of membranes.

\section{COMPUTER SIMULATION}

\section{Random Distribution of Particles by Monte} Carlo Method

The process of forming porous polymeric membranes can be investigated by computer simulation using the Monte Carlo method. In this approach, polymer particles and vacant particles, whose number ratio is predetermined by two-phase volume ratio $R$, are assumed to have the same radius of $S_{2}$. Particles are arranged randomly on a hypothetical hexagonally close-packed lattice plane and all pores formed in the plane are counted one by one to give number of vacant-particle pores with $x$ vacant particles in unit area of membrane, $N_{\mathrm{P}}(x)$ and number of vacant-particle pores in unit area of membrane (i.e., pore density of vacant-particle pores), $N_{\mathbf{P}}$ ( $\equiv \sum_{x=1}^{\infty} N_{\mathbf{P}}(x)$. By repeating random arrangements under the given condition of $R$ and $S_{2}$, probability of appearance of $N_{\mathrm{P}}$ vacant-particle pores in unit area of membrane (i.e., frequency distribution of $\left.N_{\mathrm{P}}\right), Q\left(N_{\mathrm{P}}\right)$ and that of appearance of $N_{\mathrm{P}}(x)$ vacant-particle pores consisting of $x$ vacant particles in unit area of membrane, $Q\left(N_{\mathrm{P}}(x)\right)$ were obtained. Then, expected value of $N_{\mathrm{P}}, \overline{N_{\mathrm{P}}}$ (i.e., $N_{\mathbf{P}}$ by simulation) and that of number of vacant-particle pores with $x$ vacant particles in unit area, $\overline{N_{\mathrm{p}}(x)}$ were calculated from $Q\left(N_{\mathrm{P}}\right)$ and $Q\left(N_{\mathrm{P}}(x)\right)$, respectively. Based on $\overline{N_{\mathrm{P}}(x)}$ and $\overline{N_{\mathrm{P}}}$, the probability $P(x)\left(\equiv \overline{N_{\mathrm{P}}(x)} / \overline{N_{\mathrm{P}}}\right)$ that a given pore contains $x$ vacant particles (hereafter, referred to as "pore size distribution" $P(x)$, if no confusion). If necessary, by assuming circular pore, $P(x)$ can be converted into the $N(r)$. The average of number of vacant particles in one pore $\bar{x}$ was also determined.

Figure 2 shows the flow chart of the simulation. Note that in the simulation the occupancy of a given site is approximated by the overall fraction of occupied lattice sites (i.e., $R$ ). The calculations were performed using personal computer model PC-9801 RX (manufactured by NEC Corp., Tokyo, Japan) and engineering work station model NEWS (manufactured by SONY Corp., Tokyo, Japan).

\section{EXPERIMENTAL}

\section{Sample Preparation}

Regenerated cellulose membranes were prepared from cellulose cuprammonium solutions in the same manner as that used in the previous paper. $^{3}$

\section{Measurements of Porosity and Apparent Phase Volume Ratio $R_{\mathrm{A}}$}

Electron micrographs of the surface of regenerated cellulose membranes were taken using a field emission scanning electron microscope (FE-SEM S-800, manufactured by
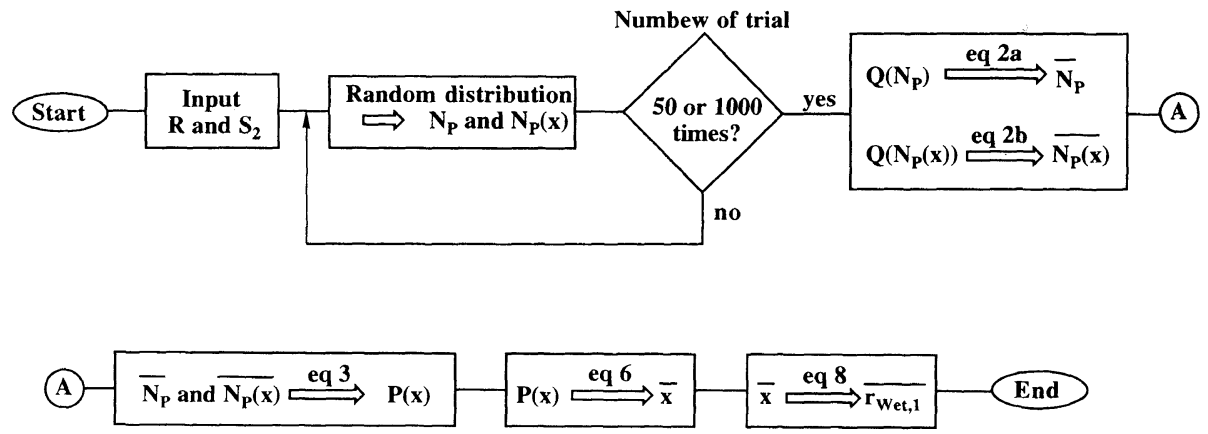

Figure 2. Flow chart of the simulation. 
Hitachi, Ltd., Tokyo, Japan). Patterns of particles in the most external surface of electron micrographs (in other words, the first single layer with a thickness of $2 S_{2}{ }^{\prime} ; S_{2}{ }^{\prime}$, radius of dry polymer particles in dry membrane) were hypothetically sliced off from the micrographs by using an image processor (model IP-1000, manufactured by Asahi Chemical Industry Co., Ltd., Tokyo, Japan) and the porosity $\operatorname{Pr}\left(\mathrm{d}_{\mathbf{4}}\right)$ of the layer was evaluated by area fraction of all pores. The porosity was converted to apparent phase ratio $R_{\mathrm{A}}$ by the relation,

a) $R=9.4$

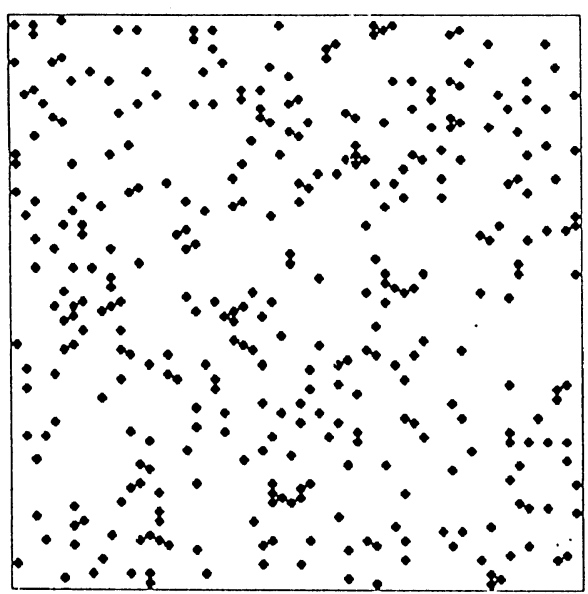

c) $R=1.0$

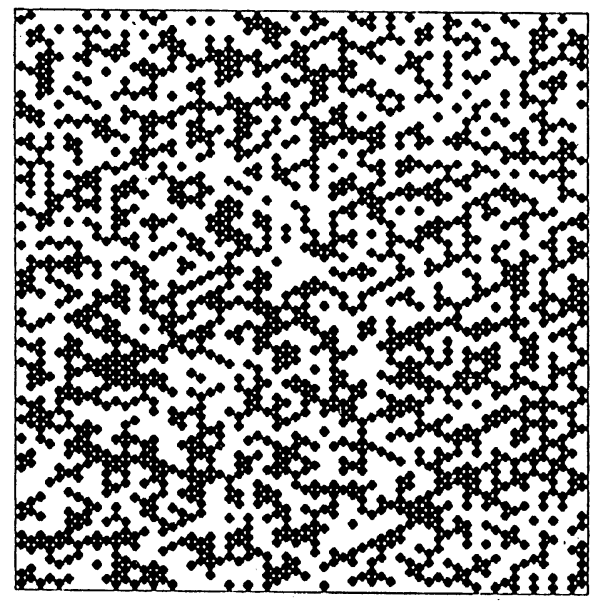

$$
R_{\mathrm{A}}=\frac{\operatorname{Pr}\left(\mathrm{d}_{4}\right)}{1-\operatorname{Pr}\left(\mathrm{d}_{4}\right)}
$$

\section{RESULTS AND DISCUSSION}

Pore Characteristics of Membranes by Random Distribution of Particles

Figure 3 shows typical examples of arrangements of polymer particles on a hypothetical plane. In this figure, vacant particles are omitted, and not shown here. In the case of

b) $R=2.3$

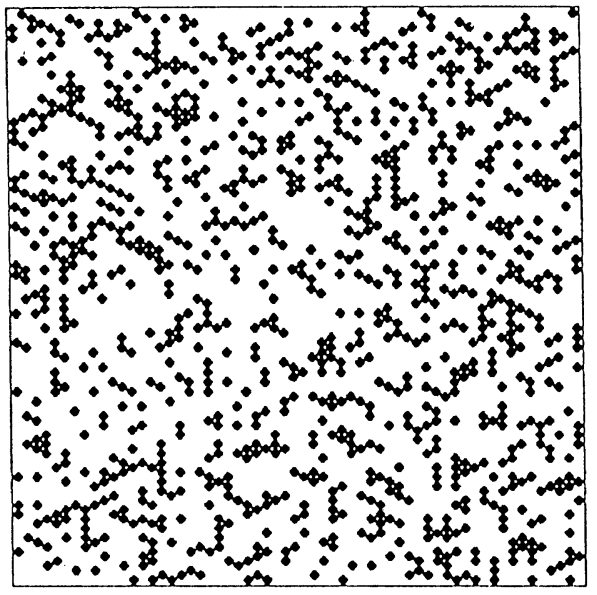

d) $R=0.43$

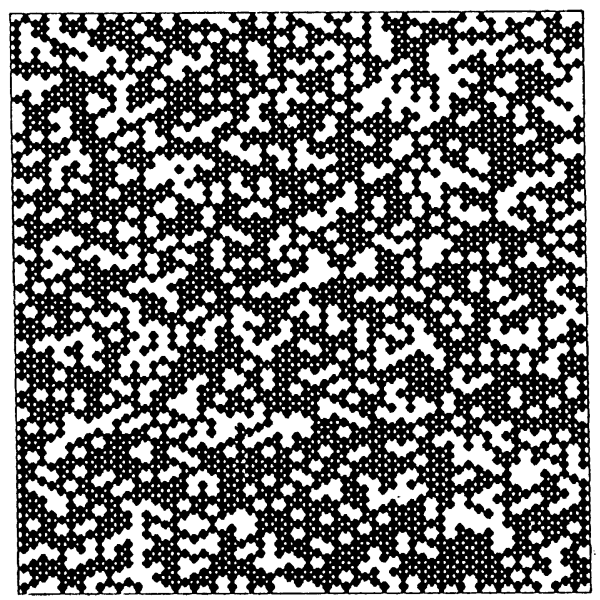

Figure 3. Typical arrangements of polymer particles (filled circles) on a hypothetical plane by computer simulations: a), Two-phase volume ratio $R, 9.4 ; \mathrm{b}), R, 2.3 ; \mathrm{c}), R, 1.0 ; \mathrm{d}), R, 0.43$. 
$R=9.4$, all polymer particles are almost completely isolated, resulting in a single large pore together with a small number of very small pores (not shown here). Experimentally similar patterns are observed by an electron microscope for polymer solutions at stage $\mathrm{f}$ in Figure $1 .^{6}$ With a decrease in $R$ (especially, at $R<1$ ), polymer particles contact each other, forming a network-like structure, and at last almost all polymer particles connect with each other, resulting in a lot of small vacantparticle pores as shown in Figure 3d.

Pore Density. In computer simulations, the total number of polymer- and vacant-particles in unit area of the plane, $N_{\mathrm{T}}$ is unambiguously determined for a given $S_{2}$ as $1 /\left(2 \sqrt{3} S_{2}{ }^{2}\right)$. Then, computer experiments are carried out under given combination of $R$ and $S_{2}$, resulting in the membrane with number of vacantparticle pores per unit area of the plane, $N_{\mathbf{P}}$ (referred to as "pore density," if no confusion). $N_{\mathrm{P}(\mathrm{v})}$ is the theoretically expected value of $N_{\mathrm{P}}$ and $N_{\mathrm{P}(\mathrm{v})}$ is unambiguously determined for given combination of $R$ and $S_{2}$ in IMK theory. ${ }^{3}$

Note that arrangements of polymer particles and vacant particles on the layer are statistical phenomena. Accordingly, $N_{\mathrm{P}}$ and number of vacant-particle pores with $x$ vacant particles in unit area of membrane, $N_{\mathrm{P}}(x)$ are determined for a given specific arrangement, in consequence, both $N_{\mathrm{P}}$ and $N_{\mathrm{P}}(x)$ have unavoidably some distributions. Mean values of $N_{\mathrm{P}}\left(\right.$ i.e., $\left.\overline{N_{\mathrm{P}}}\right)$ and those of pore density of pores with $x=1$ (i.e., $\left.\overline{N_{\mathrm{P}}(1)}\right)$ can be evaluated from their frequency distribution of $N_{\mathrm{P}}, Q\left(N_{\mathrm{P}}\right)$ and frequency distribution of $N_{\mathrm{P}}(1), Q\left(N_{\mathrm{P}}\right.$ (1)) through the relations,

$$
\overline{N_{\mathrm{P}}}=\int_{0}^{\infty} N_{\mathrm{P}} Q\left(N_{\mathrm{P}}\right) \mathrm{d} N_{\mathrm{P}}
$$

and

$$
\overline{N_{\mathrm{P}}(1)}=\int_{0}^{\infty} N_{\mathrm{P}}(1) Q\left(N_{\mathrm{P}}(1)\right) \mathrm{d} N_{\mathrm{p}}(1) .
$$

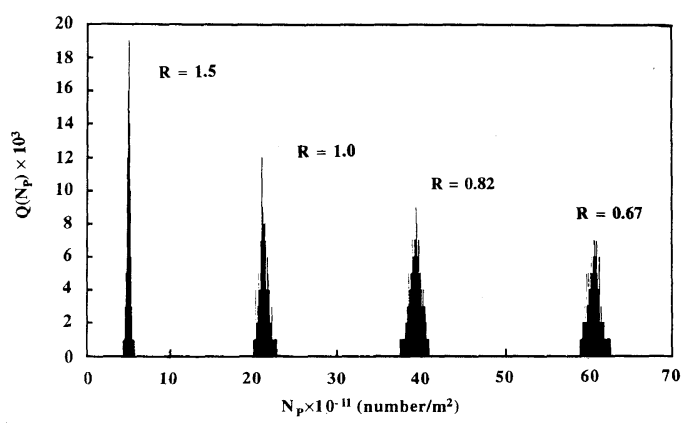

Figure 4. Frequency distribution of number of vacantparticle pores in unit area of membrane by simulation $Q\left(N_{\mathrm{P}}\right)$ for various two-phase volume ratio $R: 500 \times 500$ lattice sites; repetition of calculation (i.e., number of trials), 1000 times; $S_{2}, 50 \mathrm{~nm}$.

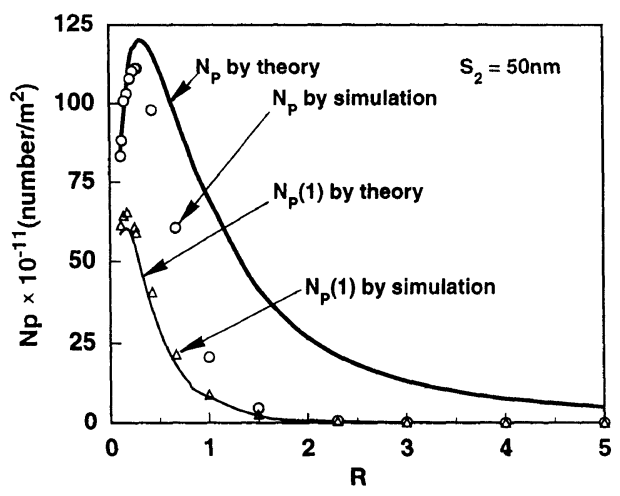

Figure 5. Effect of two-phase volume ratio $R$ on mean value of number of vacant-particle pores in unit area of membrane, $\overline{N_{\mathrm{P}}}$ (open circle; by simulation), that of the pore consisting of one vacant particle (i.e., $x=1), \overline{N_{\mathrm{P}}(1)}$ (open triangle; by simulation), number of vacant-particle pores in unit area of membrane, $N_{\mathbf{P}(v)}$ (thick full curve; by IMK theory), and that of the vacant-particle pore with $x=1, N_{\mathrm{P}(\mathrm{v})}(1)$ (thin full curve; by IMK theory); $S_{2}, 50 \mathrm{~nm}$.

Hereafter, we express $\overline{N_{\mathrm{P}}}$ and $\overline{N_{\mathrm{P}}(x)}$ simply as $N_{\mathrm{P}}$ by simulation and $N_{\mathrm{P}}(x)$ by simulation, respectively if no confusion.

Figure 4 shows $Q\left(N_{\mathrm{P}}\right)$, obtained under different two-phase volume ratio $R$ (form 0.67 to 1.5$)$. Here, $N_{\mathrm{P}}$ value was determined for a sheet of the membrane hypothetically cast on the plane of $500 \times 500$ lattice sites, and the frequency distribution of $N_{\mathrm{P}}, Q\left(N_{\mathrm{P}}\right)$ was evaluated on 1000 sheets of the membranes. In the figure, $S_{2}$ is taken as $50 \mathrm{~nm}$. In the range 

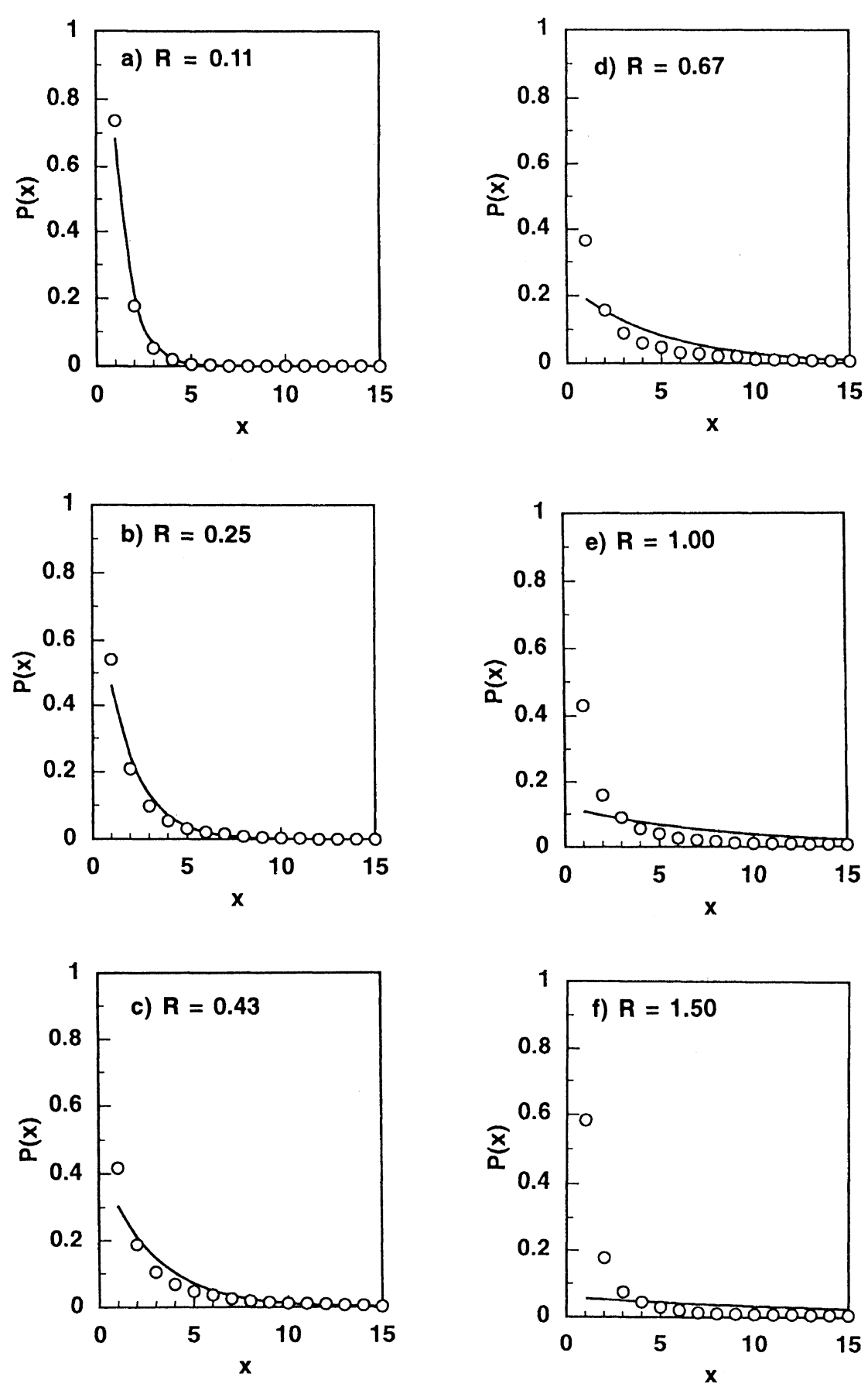

Figure 6. Comparison of the pore size distribution $P(x)$ of membrane, hypothetically cast in computer simulation experiments, (open circle) with that calculated by eq 5 of IMK theory ${ }^{3}$ (full line): a), Two-phase volume ratio $R, 0.11 ; \mathrm{b}), R, 0.25 ; \mathrm{c}), R, 0.43$; ), $R, 0.67$; ), $R, 1.00 ; \mathrm{f}), R, 1.50$. 
of $R>0.3, Q\left(N_{\mathrm{P}}\right)$ shifts to smaller $N_{\mathrm{P}}$ side and distribution becomes narrower when $R$ increases, and in the range of $R<0.3, Q\left(N_{\mathrm{P}}\right)$ shifts to larger $N_{\mathrm{P}}$ side.

Figure 5 shows effects of $R$ on $\overline{N_{\mathrm{P}}}$ (eq $2 \mathrm{a}$; open circle; $N_{\mathrm{P}}$ by simulation) and $\overline{N_{\mathrm{P}}(1)}$ (eq $2 \mathrm{~b}$; open triangle; $N_{\mathrm{P}}(1)$ by simulation), both evaluated by the computer simulations. In the figure, $N_{\mathrm{P}(\mathrm{v})}\left(N_{\mathrm{P}}\right.$ by theory) is represented by thick full curve and pore density for vacant-particle pores with $x=1, N_{\mathrm{P}(\mathrm{v})}(1)\left(N_{\mathrm{P}}(1)\right.$ by theory) is also represented by thin full curve. Here, $S_{2}$ is assumed to be $50 \mathrm{~nm}$.

Both $N_{\mathrm{P}}$ by simulation and that by IMK theory attain maximum at $R=0.3$, and $N_{\mathrm{P}}(1)$ by simulation as well as that by IMK theory show maximum at $R=0.2 . N_{\mathrm{P}}$ by simulation diminishes rapidly with an increase in $R$, approaching unity, but $N_{\mathbf{P}}$ by theory is larger than $N_{\mathrm{P}}$ by simulation and becomes smaller gradually with increasing $R . N_{\mathrm{P}}(1)$ by theory is in excellent agreement with that by simulation over the whole $R$ range. These facts mean that the IMK theory overestimates number of vacant-particle pores having $x(>1)$ vacant particles per unit area, $N_{\mathbf{p}}(x)$ with $x>1$.

By shifting the curves (by simulations) and plots (by theory) in Figure 5 to the direction of the vertical axis, relations between $N_{\mathrm{P}}$ and $R$ for given $S_{2}$ can be obtained: When $S_{2}$ increases by a factor of $10, N_{\mathrm{P}}$ for a given $R$ decreases by a factor of $1 / 100$.

Pore Size Distribution $P(x)$. Figure 6 shows pore size distribution $P(x)$ by computer simulations (open circle) which is calculated by using eq 3 for various given values of $R$ :

$$
P(x)=\frac{\overline{N_{\mathrm{P}}(x)}}{\overline{N_{\mathrm{P}}}} .
$$

Here, $\overline{N_{\mathrm{P}}}$ is defined by the relation,

$$
\overline{N_{\mathrm{P}}}=\sum_{x=1}^{\infty} \overline{N_{\mathrm{P}}(x)} \text {. }
$$

In the figure, $P(x)$, calculated using eq 5 in the IMK theory, ${ }^{3}$ is shown as a full curve for comparison:

$$
\begin{gathered}
P(x) \cong \frac{\left(1-\frac{N_{\mathrm{P}(\mathrm{v})}}{N_{\mathrm{T}} L}\right)}{\left(\frac{N_{\mathrm{T}} L}{N_{\mathrm{P}(\mathrm{v})}}-1\right)} \\
\text { for } 1 \leq x \leq N_{\mathrm{T}} L \text { and } N_{\mathrm{P}(\mathrm{v})}<N_{\mathrm{T}} L
\end{gathered}
$$

where $L$ is volume fraction of polymer-lean phase $(=R /(R+1))$.

Computer experiments indicate that $P(1)$ is the largest among $P(x)$ irrespective of $R$, i.e., the pore having a single vacant particle is the most popular. At $R \leq 0.11$, the theory is in an excellent agreement with the computer experiments over an entire range of $x$. However, in the range $R$ of $0.25-0.43$ the theory becomes slightly larger than experiments except $x=1$. With an increase in $R, P(1)$ by the theory decreases and the disparity of the theory from the simulations at $x=1$ becomes remarkably large. On the other hand, $P(x)$ by the theory exceeds those by the simulations in larger $x$ region with an increase in $R$.

Considering that the theory estimates number of vacant-particle pore consisting of one vacant particle in unit area of membrane, $N_{\mathrm{P}(\mathrm{v})}(1)\left(N_{\mathrm{P}}(1)\right.$ by theory) properly in the whole range of $R$ as shown in Figure 5, we can conclude that number of vacant-particle pores consisting of $x$ vacant particles in unit area of membrane by theory, $N_{\mathrm{P}(\mathrm{v})}(x)$ (simply expressed as $N_{\mathrm{P}}(x)$ by theory) is overestimated at $x>1$.

Figure 7 shows plots of frequency $P(1)$ as a function of $R$. Three cases of simulations, illustrated by curves with open marks, and theoretical results (thick full curve; calculated by eq 5) are shown in the figure. In the range of $R<3, P(1)$ by simulation is independent of the size of the layer assumed, but in the range $R>3, P(1)$ is larger as the size of the layer is larger, that is, at larger $R$ region the effect of the edge of the lattice space in the calculation can not be neglected. In the figure, the curve calculated for $1000 \times 1000$ lattice sites can roughly be considered as an envelope line. 


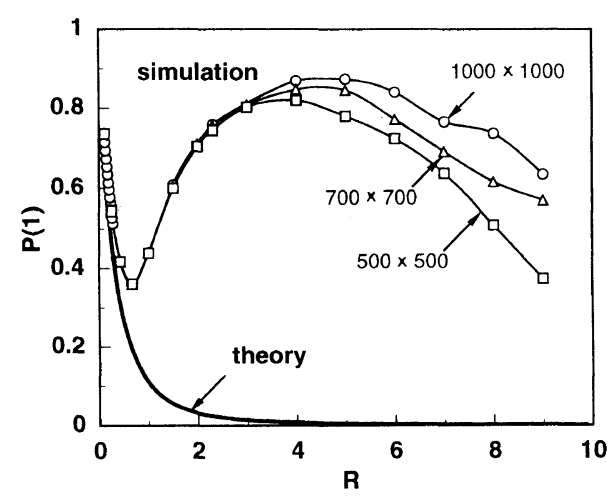

Figure 7. Frequency of vacant-particle pore consisting of a single vacant particle, $P(1)$ as a function of two-phase volume ratio $R$ : Computer simulations, open square, $500 \times 500$ lattice sites; open triangle, $700 \times 700$ lattice sites; open circle, $1000 \times 1000$ lattice sites; average of calculation (i.e., number of trials), 50 times; $P(1)$ by theoretical calculation (eq 5), thick full curve.

$P(1)$ by the simulation changes in complicated manner with $R: P(1)$ decreases first with an increase in $R$ and then increases again after passing through minimum, and then $P(1)$ exhibits the second maximum. This characteristic features of $P(1)$ obtained by the simulation are well explained by $R$ dependency of $\overline{N_{\mathrm{P}}}$ and $\overline{N_{\mathrm{P}}(1)}$ in Figure 5.

The theoretical $P(1)$ decreases rapidly and monotonously with an increase of $R$ in the whole range of $R$, becoming almost zero in the range of $R>4$. In the range of $R<0.3$, the theory coincides fairly well with the corresponding curve by the simulation. In other words, data for pore size distribution $P(x)$ of the membrane prepared at relatively large $R$ can not be fitted by the rather simple eq 5 .

In the previous paper, ${ }^{3}$ the pore characteristics of the actual dry membrane was proved to be well represented not by $R$ value evaluated directly from the phase separation experiments under the same conditions as those of membrane preparation, but by the apparent $R$ value $\left(R_{\mathrm{A}}\right)$ determined for actual membranes, using $\operatorname{Pr}\left(\mathrm{d}_{3}\right)$ and $\operatorname{Pr}\left(\mathrm{d}_{4}\right) \cdot{ }^{3}$ Those $R_{\mathrm{A}}$ of the actual membranes usually fall in the range less than unity and the theory is in a good

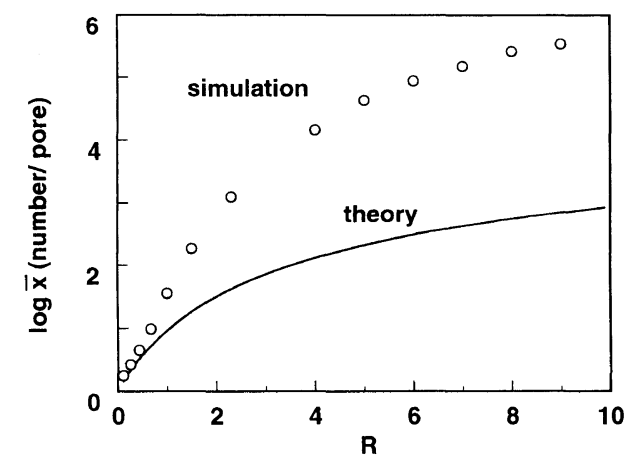

Figure 8. Comparisons between mean number of vacant particles in a pore, $\bar{x}$ by theory and that by computer simulations: Open circle, simulation; layer space, $1000 \times$ 1000 lattice sites; repetition of calculation (i.e., number of trials), 50 times; $S_{2}, 50 \mathrm{~nm} ;\left(R, \overline{N_{\mathrm{p}}}\right),\left(9.0,3.79 \times 10^{8}\right.$ number $\left./ \mathrm{m}^{2}\right), \quad\left(8.0,4.92 \times 10^{8}\right), \quad\left(7.0,8.48 \times 10^{8}\right), \quad(6.0$, $\left.1.43 \times 10^{9}\right),\left(5.0,2.86 \times 10^{9}\right),\left(4.0,8.11 \times 10^{9}\right),(2.3,8.23 \times$ $\left.10^{10}\right),\left(1.5,4.80 \times 10^{11}\right),\left(1.0,2.08 \times 10^{12}\right),(0.667,6.04 \times$ $\left.10^{12}\right), \quad\left(0.429, \quad 9.79 \times 10^{12}\right), \quad\left(0.25, \quad 1.11 \times 10^{13}\right), \quad(0.11$, $8.31 \times 10^{12}$ ); thick full curve, theory (eq 7); $S_{2}, 50 \mathrm{~nm}$.

agreement with the simulation only at small $R$ values $(0.1-0.4)$ and then, the theory holds its validity in a practical use.

Mean Pore Size. The average of number of vacant particles $x$ in a single pore, $\bar{x}$ is defined by

$$
\bar{x}=\int_{1}^{\infty} x P(x) \mathrm{d} x .
$$

$\bar{x}$ thus estimated by the simulation is plotted against $R$ as open circle in Figure 8. In addition, for a given combination of $R$ and $S_{2}$ (accordingly, $L), \bar{x}$ is also calculated from the relation in the IMK theory ${ }^{3}$;

$$
\bar{x}=\frac{N_{\mathrm{T}} L}{N_{\mathrm{P}(\mathrm{v})}} .
$$

Here, note that $N_{\mathrm{P}(\mathrm{v})}$ is determined from $S_{2}$ and $R$ and is reversely proportional to $S_{2}{ }^{2}$ (see, eq 29 of ref 3 ) and the ratio $N_{\mathrm{T}} / N_{\mathrm{P}(\mathrm{v})}$ is a single function of $R$. Equation 7 shows that $\bar{x}$ is independent of $S_{2}$. The results on $\bar{x}$ evaluated by eq 7 are also included in the figure as a full curve. Both the simulation and the theory show that $\bar{x}$ gets larger with an increase in $R$ and in 
particular the effect of $R$ on $\bar{x}$ is remarkable in the small $R$ region. In the range $0<R<0.3, \bar{x}$ by the theory is in excellent agreement with those by the simulation and even in the range $0.3<R<0.5$, the theory agrees fairly well with the simulation. As the theory overestimates $N_{\mathrm{P}}$ in larger $R$ region, disparity of the theory from the simulation becomes larger in the region of $R>0.5$.

Figure 9 shows the $R$ dependence of the ratio of the number-average pore radius for wet membrane to $S_{2}, r_{\text {Wet, } 1} / S_{2}$, which is calculated

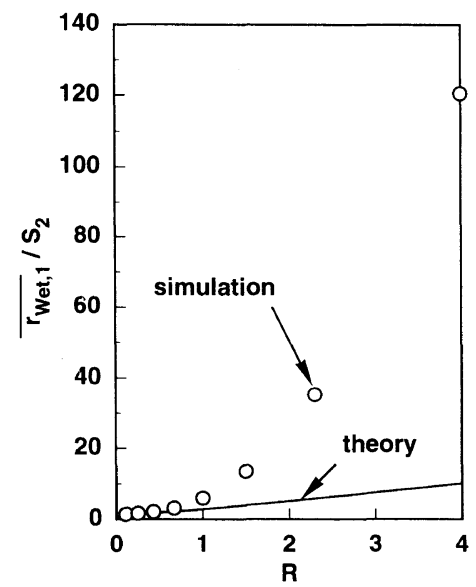

Figure 9. Relationship between ratio $\overline{r_{\mathrm{Wet}, 1}} / S_{2}$ and two-phase volume ratio $R: \overline{r_{\text {Wet } 1}}$, mean pore radius of wet membrane; $S_{2}$, radius of polymer particle; open circle, computer simulations (eq 6 and 8); circular pore growth was assumed; full line, theory (eq 7 and 8). by using eq 6 and 8 in the simulation (open circle) or by using eq 7 and 8 in the theory (full line);

$$
\overline{r_{\mathrm{Wet}, 1}}=\sqrt{\bar{x}} S_{2} .
$$

Note that the ratio $r_{\mathrm{Wet}, 1} / S_{2}$ is a single function of $R . \overline{r_{\mathrm{Wet}, 1}}$ increases with $R$ and its rate of increase $\mathrm{d} \overline{r_{\mathrm{Wet}, 1}} / \mathrm{d} R$ is larger for larger $S_{2}$.

\section{Validity of $P_{n}(m)$ Values of IMK Theory}

In the IMK theory, a polymer particle on the lattice can participate at most the formation of three different pores (see, Figure 8 of ref 3 ). When a single polymer particle is directly participated to the formation of $n$ vacantparticle pores and $m$ vacant particles are existing around the particle, (i.e., $m$ is the number of the nearest neighbor vacant particles; $1 \leq m \leq 5$ ), the probability that a given polymer particle is surrounded in part by. $m$ vacant particles belonging to $n$ different vacant pores is defined as $P_{n}(m),{ }^{3}$ which is given by the relations,

$$
\begin{aligned}
& P_{0}(0)=1 /(1+R)^{6}, \\
& P_{1}(m)=6 R^{m} /(1+R)^{6} \\
& \quad \text { for } 1 \leq m \leq 5, \\
& P_{2}(2)=9 R^{2} /(1+R)^{6}, \\
& P_{2}(3)=12 R^{3} /(1+R)^{6},
\end{aligned}
$$

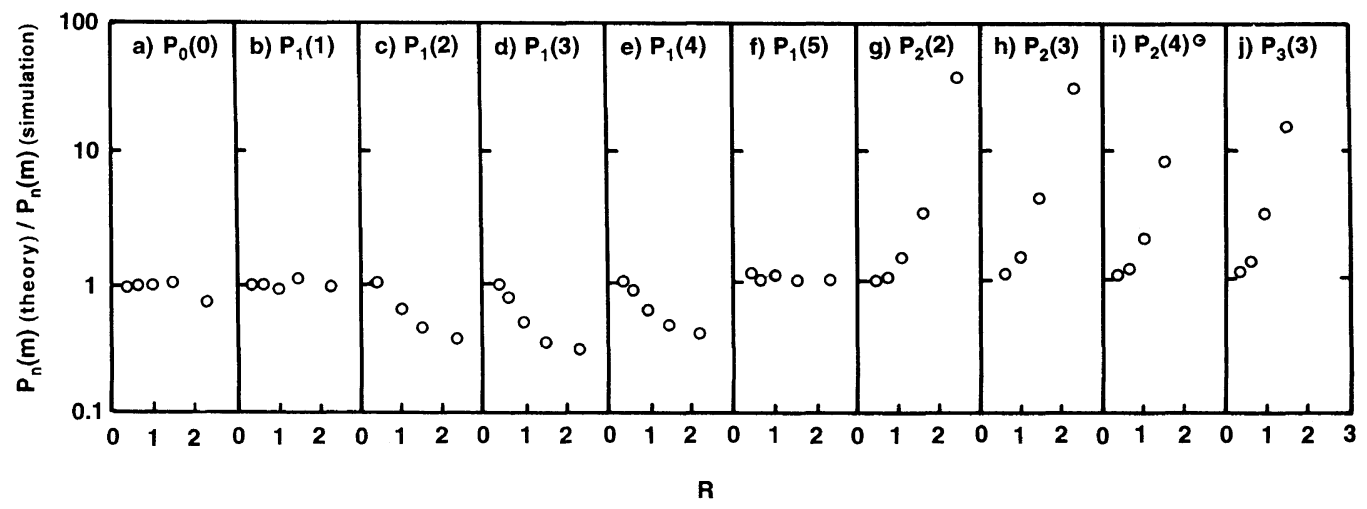

Figure 10. The ratio of $P_{n}(m)$, estimated by eq $9 \mathrm{a}-9 \mathrm{f}$ of the theory to $P_{n}(m)$, determined by the computer simulations. 


$$
\begin{aligned}
& P_{2}(4)=9 R^{4} /(1+R)^{6}, \\
& P_{3}(3)=2 R^{3} /(1+R)^{6} .
\end{aligned}
$$

Using the data by the computer simulations, we can check the validity of $P_{n}(m)$ values estimated by eq $9 \mathrm{a}-9 \mathrm{f}$.

Figure 10 shows the ratio of $P_{\mathrm{n}}(m)$ estimated by the theory (eq $9 \mathrm{a}-9 \mathrm{f}$ ) to that of computer simulations, $P_{\mathrm{n}}(m)$ (theory) $/ P_{n}(m)$ (simulation), as a function of $R$ for a given combination of $m$ and $n$. In the range of $R<2.3$ (i.e., $L<0.3$ ), $P_{0}(0)$ defined by eq 9 a coincides excellently with the simulations. Deviation of the ratio $P_{0}(0)$ (theory) $/ P_{0}(0)$ (simulation), observed at $R=$ 2.3 , from unity may be caused by a large scattering of data inhering to simulation experiments at higher $R$. The ratio corresponding to $P_{1}(m)(m=2,3$, and 4$)$ deviates from unity, increasing with an increase in $R$. The striking deviation of the theory from the simulations for $m \geqq 2$ is noticed at $R \geqq 0.2$. In the theory, an arrangement, as illustrated in Figure $11(m=4)$, is treated as a case of $P_{2}(4)$ (i.e., a given polymer particle (filled circle) are considered to participate the formation of two different pores), but in the computer simulations all the arrangements of polymer particles and vacant particles in a plane are completely considered in calculating any $P_{n}(m)$, and then some portion of $P_{2}(4)$ in the theory may be

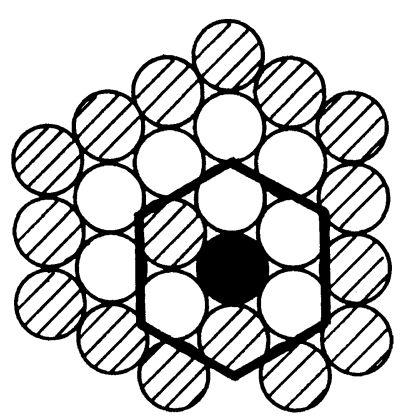

Figure 11. An example of arrangements around a given polymer particle: Filled circle, a given polymer particle; hatched circle, polymer particles except the given polymer particle; unfilled circle, vacant particle; inside of a hexagon, nearest neighbor area for the given polymer particle. rightly counted by the simulation as a case of $P_{1}(4)$, when four vacant particles are connected indirectly outside the nearest neighbor area of the given polymer particles as shown in Figure 11. This indicates that the theory leads often to underestimation of $P_{1}(m)(m=2,3$, and 4), especially in the larger $R$ region. It is proper that the ratios of $P_{1}(m)(m=1$ and 5) are always unity over a wide range of $R$. In the range of $R \leqq 0.3, P_{2}(m)(m=2,3$, and 4$)$ and $P_{3}(3)$, calculated by eq $9 \mathrm{c}-9 \mathrm{f}$, agree well with those of the simulations. The ratios of the above $P_{n}(m)$ deviate remarkably from unity as $R$ increases beyond 0.67 , because $P_{2}(m)$ and $P_{3}(3)$ are significantly overestimated in the theory, in which only the nearest neighbors are taken into account. Therefore, we can conclude that in the larger $R$ region, $P_{n}(m)$ value by the theory deviates significantly from that by the simulations. This means that the theory becomes unavoidably crude at larger $R$.

\section{Effect of Piling of Planes}

Figure 12 shows some arrangements of polymer particles when a given number $\left(n_{\mathrm{P}}\right)$ of hypothetical thin gel planes are piled up to amalgamate or to melt down to give a single gel plane, whose thickness is almost equivalent to the diameter of the polymer particles. In this case, apparent phase volume ratio $R_{\mathrm{A}}$ is defined by the relation, ${ }^{3}$

$$
R_{\mathrm{A}}=\frac{\left(\frac{R}{R+1}\right)^{n_{\mathrm{P}}}}{1-\left(\frac{R}{R+1}\right)^{n_{\mathrm{P}}}} .
$$

In deriving eq 10, an overlapping of polymer particles belonging to different hypothetical gel planes was assumed to be totally absent. Computer simulations show that this approximation of non-overlapping is not too crude: When 10 gel planes formed at $R=9.0$ are piled and collapsed in the simulation, the phase volume ratio evaluated by taking into consideration overlapping for the piled planes, $R$ is 
a) $R=9.1$ and $n_{\mathrm{p}}=1$

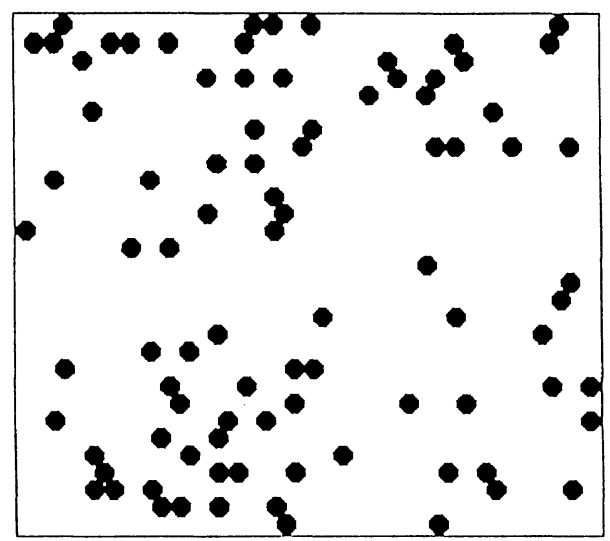

b) $R=9.0$ and $n_{\mathrm{P}}=10 ; R_{\mathrm{A}}=0.53$

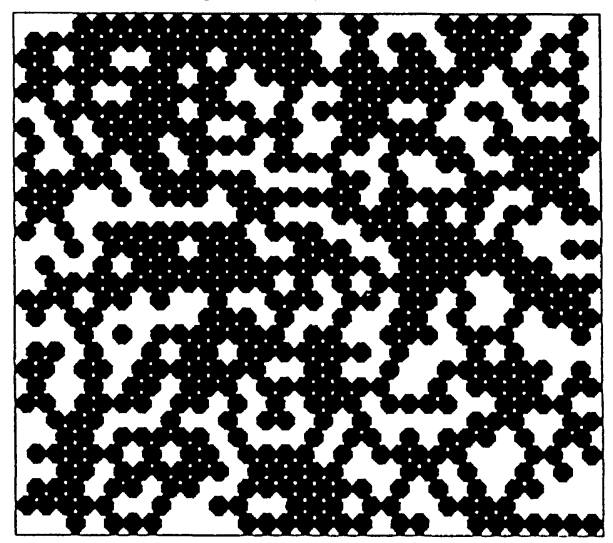

c) $R=0.54$ and $n_{\mathrm{P}}=1$

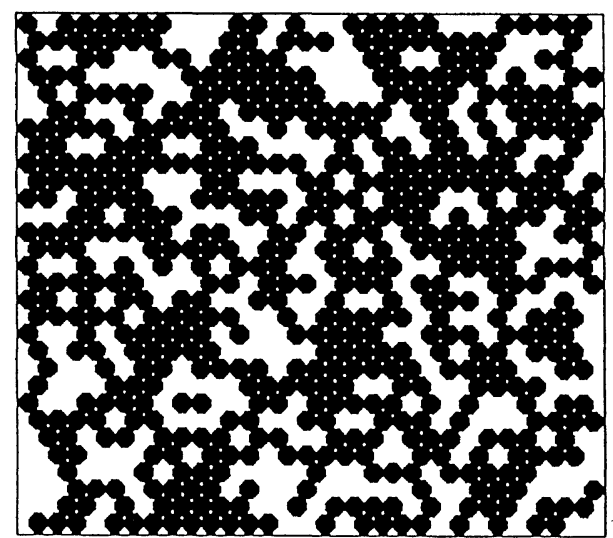

Figure 12. Morphology of hypothetical thin planes which are made by piling up a given number $\left(n_{\mathrm{p}}\right)$ of hypothetical gel layers formed at common $R$ by computer simulations: $R_{\mathrm{A}}$, eq $\left.10 ; \mathrm{a}\right), R=9.1$ and $\left.n_{\mathrm{P}}=1 ; \mathrm{b}\right), R=9.0$ and $\left.n_{\mathrm{P}}=10 ; R_{\mathrm{A}}, 0.53 ; \mathrm{c}\right), R=0.54$ and $n_{\mathrm{P}}=1$.

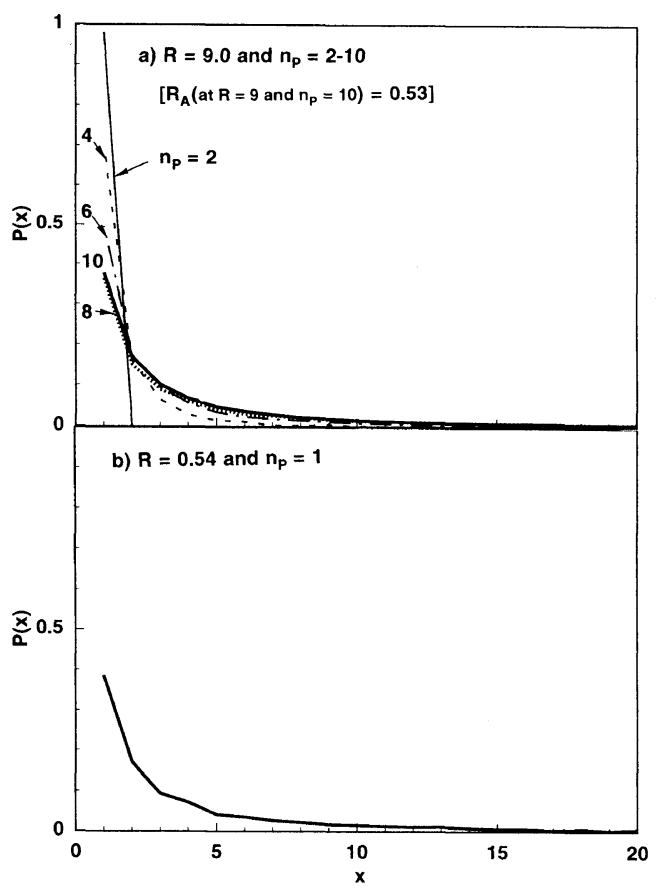

Figure 13. Pore size distribution $P(x)$ of hypothetical thin membrane made by piling $n_{\mathrm{P}}$ hypothetical gel planes formed at common $R$ by computer simulations; a), Changes of $P(x)$ depending on the number of piling from $n_{\mathrm{P}}=2$ to 10 in a case of $R=9.0 ; R_{\mathrm{A}}$ (at $R=9.0$ and $n_{\mathrm{P}}=10$ ), 0.53; b), $R=0.54$ and $n_{\mathrm{P}}=1$.

0.53 , which should be compared with $R_{\mathrm{A}}=$ 0.54 , calculated by assuming non-overlapping (eq 10). Figure 12c shows morphology of a hypothetical thin plane directly formed at $R=0.54$. This can be compared with that of the plane formed by compilation of ten thin planes with $R=9.0$ (Figure 12b). Morphology of these two planes is absolutely indiscriminate.

Figure 13a shows the changes of the pore size distribution $P(x)$ of thin gel membrane prepared by piling one to ten gel membranes at $R=9.0$. As the number of piling $\left(n_{\mathrm{P}}\right)$ is increasing, the value of $P(1)$ is becoming smaller and smaller. $R_{\mathrm{A}}$ of the membrane prepared by piling ten gel planes was 0.53 . Figure $13 \mathrm{~b}$ shows $P(x)$ of a membrane made directly at $R=0.54$. $P(x)$ of these membranes is practically the same. 
Comparison of Morphology with Actual Membranes

Figure 14 shows comparisons of morphology of hypothetical thin membranes made by the simulations with that of regenerated cellulose membrane with corresponding apparent phase volume ratio $R_{\mathrm{A}}$. In Figures $14 \mathrm{~b}$ and $14 \mathrm{~d}$, only the first single layer with a thickness of dry plane, $2 S_{2}^{\prime}\left(S_{2}^{\prime}\right.$ is a radius of dry polymer particle) was processed by an image analyzer to be hypothetically sliced off from electron micrographs of cellulose membranes. It has been ascertained that simulations can produce membranes with morphology very similar to actual membranes.

These findings indicate explicitly that we can a) $R=7.5, n_{\mathrm{P}}=1$ (simulation)

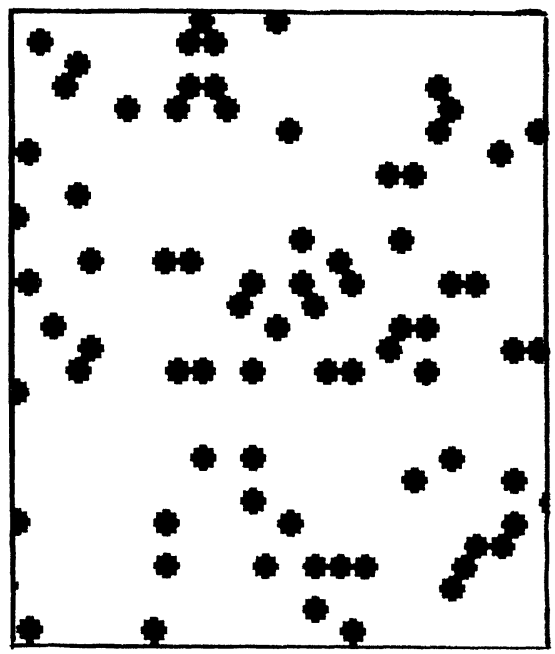

c) $R=1.1, n_{\mathrm{P}}=1$ (simulation)

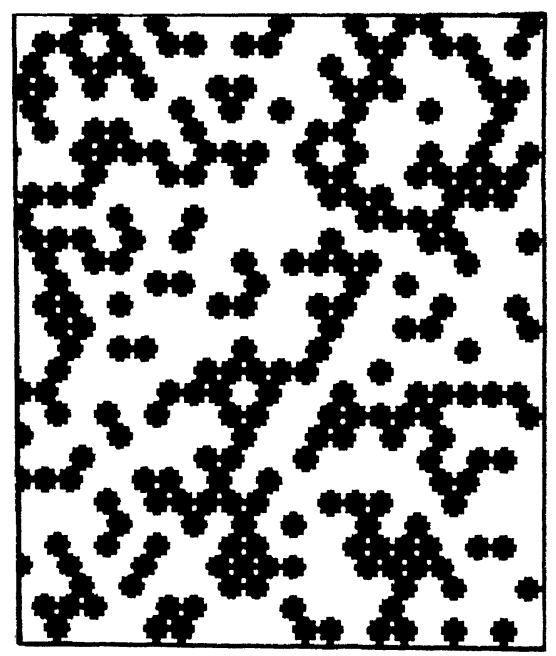

b) $R_{\mathrm{A}}=7.3$ (cellulose membrane)

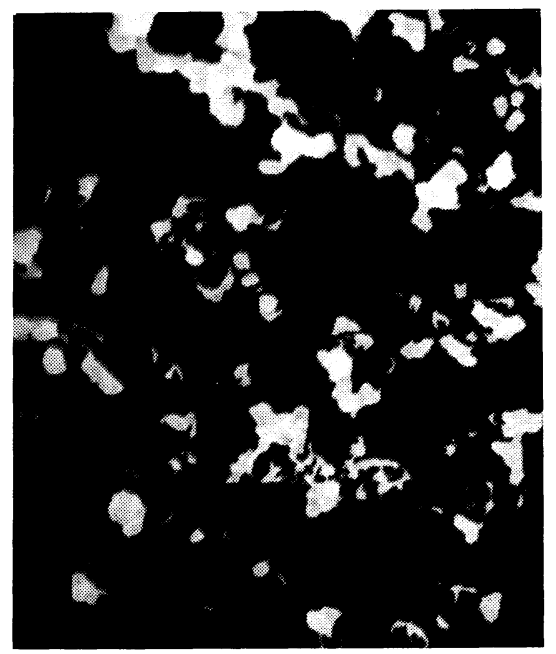

d) $R_{\mathrm{A}}=1.4$ (cellulose membrane)

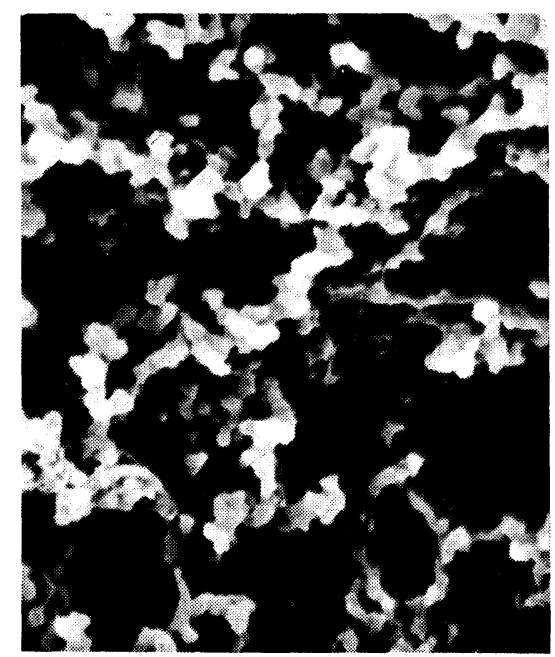

Figure 14. Morphology of hypothetical thin membranes made by computer simulations and those of regenerated cellulose membranes: a), $R=7.5$ and $n_{\mathrm{P}}=1$; computer simulation; b), $R_{\mathrm{A}}, 7.3$; regenerated cellulose membrane; $c), R=1.1$ and $n_{\mathrm{P}}=1$; computer simulation; d), $R_{\mathrm{A}}, 1.4$; regenerated cellulose membrane. 
accurately evaluate the morphology and pore size distribution by computer simulations using $R_{\mathrm{A}}$, in place of $R$ (which is measured at the casting process). Note that in the phase separation stage of $v_{\mathrm{p}}^{0}<v_{\mathrm{p}}^{\mathrm{c}}\left(v_{\mathrm{p}}^{0}\right.$, polymer volume fraction of the solution when the phase separation occurs; $v_{\mathrm{p}}^{\mathrm{c}}$, polymer volume fraction at critical solution point), $R$ should be larger than unity, but $R$ for dry membranes is usually less than unity, because some tens of hypothetical gel planes are piled up to give a single dry plane.

\section{CONCLUSIONS}

Computer simulation experiments on membrane formation lead to the conclusions as follows:

(1) Single supermolecular structure can be formed only at $R \leq 1$.

(2) Pore density $N_{\mathrm{P}}$ has unavoidably distribution and as $R$ increases, average $N_{\mathrm{P}}\left(\overline{N_{\mathrm{P}}}\right)$ is smaller and the frequency distribution of $N_{\mathrm{P}}$, $Q\left(N_{\mathrm{P}}\right)$ becomes sharp.

(3) $\quad N_{\mathrm{P}}$ attains maximum at specific $R$ and in the range $R \geq 0.3$, the theory by Iijima, Matsuda and Kamide (IMK) overestimates $N_{\mathrm{p}}$.

(4) If $R \leq 0.3-0.4$, the IMK theory gives the same $P(x)$ curve as that by the simulation, but at $R>0.5$, the $P(x)$ predicted by the theory deviates significantly from the simulation, yielding overestimated breadth in $P(x)$ curve.

(5) Except for $R \leq 0.5$, the IMK theory underestimates the average $x(\bar{x}$, accordingly, $\left.\overline{r_{\text {Wet }, 1}}\right)$.

(6) Both the theory and simulation give the same $P_{0}(0)$ and $P_{1}(1)$. The theory underestimates $P_{1}(m) \quad(m=2-4)$ and overestimates $P_{2}(m)(m=2-4)$ at larger $R$.

(7) The value of $R$, estimated from the porosity of membrane, deviates markedly from the $R$ value, which is measured directly under the same conditions as those of membrane formation. This large disparity can be explained as due to the collapse of gel layers and

subsequent piling-up of the collapsed layers. Therefore, membrane in actual use can be approximately regarded as the membrane which is produced by single step under $R \leq 1$.

(8) $P(x)$ does not change significantly when the number of gel layers piled up to make a thin layer, $n_{\mathrm{P}}$ is above $c a$. $8-10$.

(9) Computer simulation can reproduce the morphology of actual membranes.

\section{GLOSSARY OF SYMBOLS}

$L$

$N_{\mathbf{P}}$

$\overline{N_{\mathrm{P}}}$

$N_{\mathrm{P}}$

$N_{\mathrm{P}(\mathrm{v})}$

$N_{\mathrm{P}(\mathrm{v})}(x)$

$N_{\mathrm{P}}(x)$

$\overline{N_{\mathrm{P}}(x)}$

$N(r)$

$N(r)_{\mathrm{EM}}$

$N_{\mathrm{T}}$

$P_{n}(m)$

$\operatorname{Pr}\left(\mathrm{d}_{3}\right)$

$\operatorname{Pr}\left(\mathrm{d}_{4}\right)$ volume fraction of polymer leanphase $(=R /(R+1))$

number of vacant-particle pores in unit area of membrane (i.e., pore density of vacant-particle pores), $\left(\equiv \sum_{x=1}^{\infty} N_{\mathrm{P}}(x)\right)$

expected value of $N_{\mathrm{P}}$ for a given combination of $R$ and $S_{2}$ under the random distribution of particles by simulation; $N_{\mathrm{P}}$ by simulation

$N_{\mathrm{P}}$ theoretically expected for a given combination of $R$ and $S_{2}$ under the random distribution of particles number of vacant-particle pores consisting of $x$ vacant particle in unit area of membrane by theory; $N_{\mathrm{P}}(x)$ by theory

number of vacant-particle pores with $x$ vacant particles in unit area of membrane

$N_{\mathrm{P}}(x)$ by simulation

pore size distribution

$N(r)$ experimentally determined by the electron micrographic method total number of polymer- and vacant-particles in unit area of the plane

probability that a given polymer particle is surrounded in part by $m$ vacant particles, which belong to $n$ different pores

porosity determined by the apparent density method

porosity of dry membrane deter- 


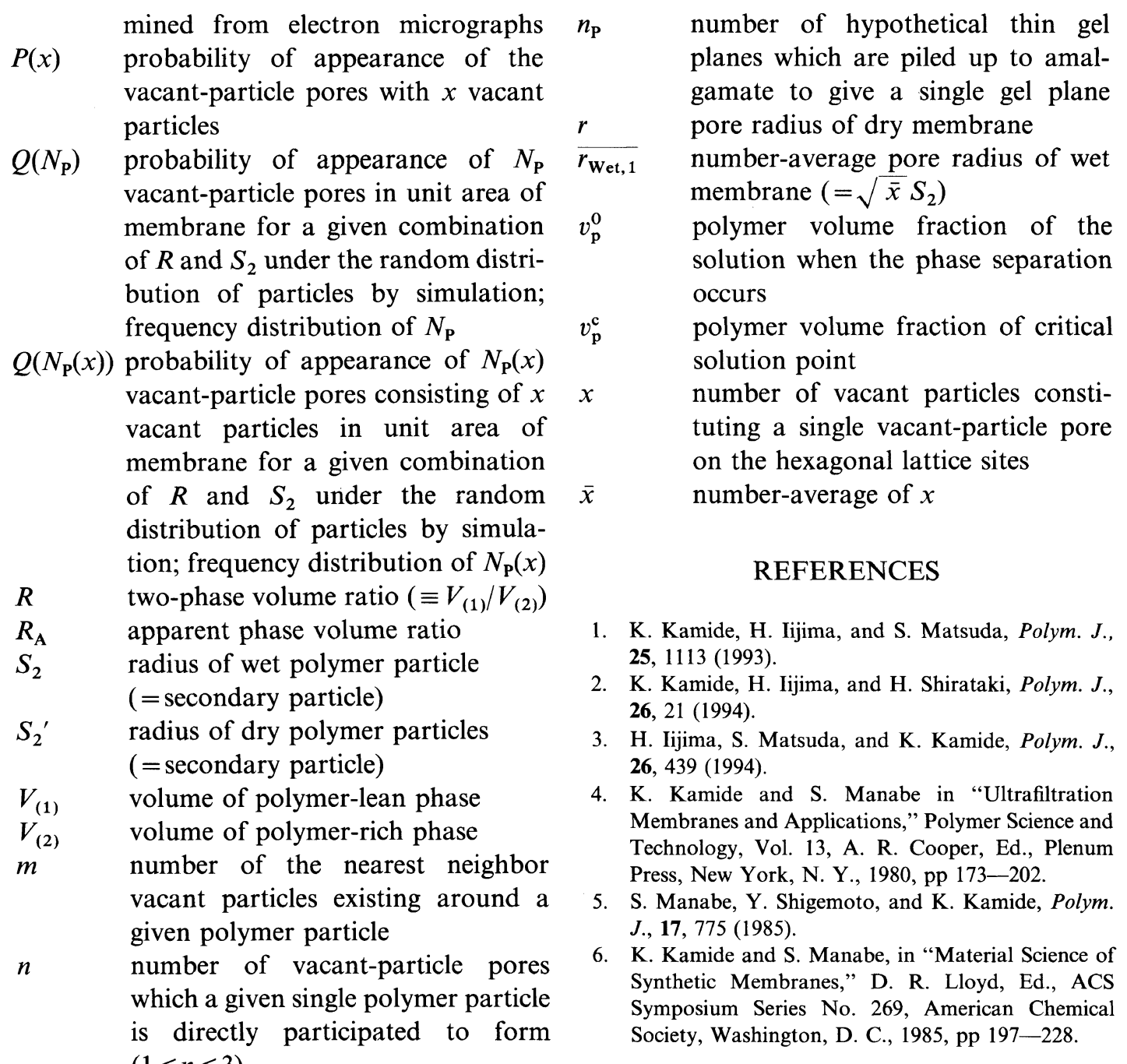
$(1 \leq n \leq 3)$ 